
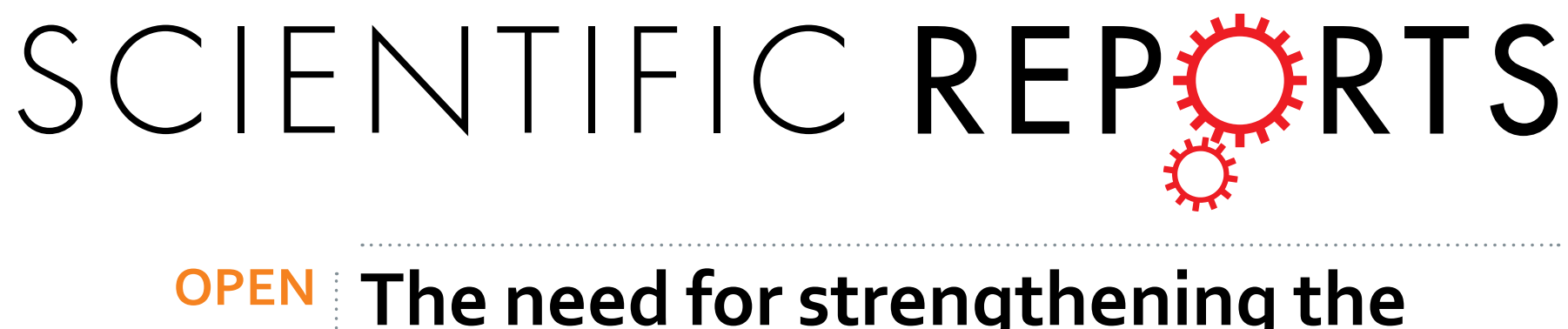

\title{
The need for strengthening the influenza virus detection ability of hospital clinical laboratories: an
}

Received: 25 October 2016

Accepted: 24 January 2017

Published: 10 March 2017 \section{investigation of the 2009 pandemic}

\author{
Shigui Yang ${ }^{1}$, Yuqing Zhou ${ }^{1}$, Yuanxia Cui ${ }^{1}$, Cheng Ding ${ }^{1}$, Jie Wu ${ }^{1}$, Min Deng ${ }^{1}$, \\ Chencheng Wang ${ }^{1}$, Xiaoqing $\mathrm{Lu}^{1}$, Xiaoxiao Chen ${ }^{1}$, Yiping $\mathrm{Li}^{2}$, Dongyan $\mathrm{Shi}^{1}$, \\ Fenfang $\mathrm{Mi}^{3}$ \& Lanjuan $\mathrm{Li}^{1}$
}

Most hospital clinical laboratories (HCLs) in China are unable to perform influenza virus detection. It remains unclear whether the influenza detection ability of $\mathrm{HCLs}$ influences the early identification and mortality rate of influenza. A total of 739 hospitalized patients with 2009 influenza A (H1N1) virus treated at 65 hospitals between May and December, 2009, in Zhejiang, China, were included based on identifications by HCLs and by public health laboratories (PHLs) of the Centers for Disease Control and Prevention. Of the patients, $407(55.1 \%)$ were male, 17 died, resulting in an in-hospital mortality rate of $2.3 \%$, and 297 patients were identified by HCLs and 442 by PHLs. The results indicated that a 24hour delay in identification led to a $13 \%$ increase in the odds of death $(O R=1.13, P<0.05)$. The time between onset and identification ( 3.9 days) of the $\mathrm{HCL}$ cohort was significantly shorter than that of the $\mathrm{PHL}$ cohort (4.8 days). The in-hospital mortality rate of the $\mathrm{HCL}$ group was significantly lower than that of the PHL group (1.0\% vs. 3.2\%, P $<0.05)$. HCL-based detection decreased the in-hospital mortality rate by $68.8 \%$. HCL-based influenza virus detection facilitated early identification and reduced influenza mortality, and influenza detection ability of HCLs should be strengthened.

After pH1N1 virus was first identified in April 2009, it spread rapidly to almost all countries during 2009 and $2010^{1-5}$. As of March 2010, more than 17,700 deaths among laboratory-confirmed cases had been reported to the World Health Organization (WHO) ${ }^{6}$. The pH1N1 influenza epidemic, though it has ended, has raised significant concerns about how to reduce the incidence of complications in influenza patients and prevent them from developing critical cases of influenza and dying.

Global efforts to enhance early disease detection and increase diagnostic abilities have stimulated the formation of laboratory networks of influenza detection and treatment ${ }^{7}$. A variety of sophisticated detection methods of pH1N1 influenza, such as RT-PCR and rtRT-PCR, were recommended by the WHO ${ }^{8}$. However, most hospital clinical laboratories (HCLs) are still unable to carry out clinical influenza virus detection, and the majority of pH1N1 influenza patients were identified by public health laboratories (PHLs) based in institutes of the Centers for Disease Control and Prevention during the pandemic ${ }^{9}$.

Currently, it remains unclear whether the ability of HCLs to detect the influenza virus influences early case identification and antiviral therapy and in particular the mortality rate of influenza. During the pH1N1 influenza pandemic in 2009, we developed a cohort of 739 hospitalized patients whose infection was identified by different types of laboratories, which enabled us to assess the influence of the HCL influenza virus detection ability on early case identification and mortality rate. 


\begin{tabular}{|c|c|c|c|c|}
\hline Variables & $\begin{array}{l}\text { Patients with PHLs based } \\
\text { detection }(\mathrm{n}=442)^{*}\end{array}$ & $\begin{array}{c}\text { Patients with HCLs based } \\
\text { detection }(n=297)\end{array}$ & $\begin{array}{l}\text { Attributable } \\
\text { fraction }(\%)^{\dagger}\end{array}$ & Pvalue \\
\hline \multicolumn{5}{|l|}{ Base conditions } \\
\hline Gender-male n (\%) & $233(52.7)$ & $174(58.6)$ & Null & 0.115 \\
\hline Age 0- & $110(24.9)$ & $84(28.5)$ & \multirow{3}{*}{ Null } & \multirow{3}{*}{0.460} \\
\hline $14-$ & $295(66.7)$ & $191(64.7)$ & & \\
\hline $60-$ & $37(8.4)$ & $20(6.8)$ & & \\
\hline $\mathrm{BMI}<18.5$ & $108(27.4)$ & $74(27.1)$ & \multirow{4}{*}{ Null } & \multirow{4}{*}{0.316} \\
\hline $18.5-$ & $187(47.5)$ & $146(53.5)$ & & \\
\hline $25-$ & $68(17.3)$ & $37(13.6)$ & & \\
\hline $30-$ & $31(7.9)$ & $16(5.9)$ & & \\
\hline Pulmonary disease $\mathrm{n}(\%)$ & $39(9.4)$ & $23(7.9)$ & Null & 0.479 \\
\hline Cardiovascular disease $\mathrm{n}(\%)$ & $55(13.3)$ & $30(10.2)$ & Null & 0.221 \\
\hline Metabolic disease n (\%) & $33(7.9)$ & $13(4.4)$ & Null & 0.056 \\
\hline Renal disease $\mathrm{n}(\%)$ & $12(2.9)$ & $12(4.1)$ & Null & 0.401 \\
\hline Liver disease $\mathrm{n}(\%)$ & $33(7.9)$ & $16(5.4)$ & Null & 0.192 \\
\hline Cancers n (\%) & $7(1.7)$ & $9(3.1)$ & Null & 0.229 \\
\hline Immunosuppression disease n (\%) & $4(1.0)$ & $7(2.4)$ & Null & 0.138 \\
\hline Nervous system disease $\mathrm{n}(\%)$ & $6(1.4)$ & $7(2.4)$ & Null & 0.365 \\
\hline \multicolumn{5}{|l|}{ Affected elements } \\
\hline $\begin{array}{l}\text { Frequency of visiting to hospital (Times, } \\
\text { mean } \pm \text { SD) }\end{array}$ & $1.8 \pm 1.2$ & $1.5 \pm 0.9$ & $16.7 \%$ & 0.004 \\
\hline $\begin{array}{l}\text { Time intervals from onset to first visit (Days, } \\
\text { mean } \pm \text { SD) }\end{array}$ & $2.2 \pm 3.1$ & $2.0 \pm 4.5$ & $9.1 \%$ & 0.522 \\
\hline $\begin{array}{l}\text { Time interval from onset to identification } \\
\text { (Days, mean } \pm \text { SD) }\end{array}$ & $4.8 \pm 3.2$ & $3.9 \pm 5.0$ & $18.8 \%$ & 0.003 \\
\hline $\begin{array}{l}\text { Time interval from first visit to identification } \\
\text { (Days, mean } \pm \text { SD) }\end{array}$ & $3.0 \pm 3.4$ & $2.1 \pm 2.9$ & $30.0 \%$ & $<0.001$ \\
\hline $\begin{array}{l}\text { Time interval from onset to hospital admission } \\
\text { (Days, mean } \pm \text { SD) }\end{array}$ & $3.7 \pm 3.0$ & $3.2 \pm 3.1$ & $13.5 \%$ & 0.045 \\
\hline $\begin{array}{l}\text { Time interval from onset to initiating antiviral } \\
\text { therapy (Days, mean } \pm \text { SD) }\end{array}$ & $4.9 \pm 3.1$ & $4.4 \pm 3.5$ & $10.2 \%$ & 0.173 \\
\hline Length of stay in hospital (Days, mean \pm SD) & $10.2 \pm 5.4$ & $7.8 \pm 5.7$ & $23.5 \%$ & $<0.001$ \\
\hline Course of disease (Days, mean \pm SD) & $14.7 \pm 6.8$ & $11.1 \pm 7.8$ & $24.5 \%$ & $<0.001$ \\
\hline In-hospital mortality n (\%) & $14(3.2)$ & $3(1.0)$ & $68.8 \%$ & 0.043 \\
\hline
\end{tabular}

Table 1. Time intervals between the primary medical activities and the outcomes of patients with pH1N1 influenza identified with PHL-based detection and HCL- based detection. ${ }^{\star}$ The samples were sent to other institutes for detection, such as the local Center for Disease Control and Prevention. ${ }^{\dagger}$ Attributable fraction (\%): the proportion of the decreased fraction due to HCL-based detection. Attributable fraction $(\%)=($ values in the group of patients with PHL-based detection - values in the group of patients with HCL-based detection)/values in the group of patients with PHL-based detection. ${ }^{\S}$ Frequency of hospital visits (times, mean), which means the number of times a patient visited a hospital for outpatient treatment before admission.

\section{Results}

Demographic and clinical features of patients. Among the cohort of 739 hospitalized patients, 407 $(55.1 \%)$ were male and $332(44.9 \%)$ female, and $194(26.3 \%)$ were children $(<14$ years old), $486(65.9 \%)$ were aged $14-60$, and $57(7.7 \%)$ were more than 60 years old. Forty-seven (7.0\%) had a body mass index higher than 30 , and $43(6.0 \%)$ were pregnant. In the cohort, 619 patients had a fever, and 578 had a cough, accounting for $98.3 \%$ and $96.5 \%$ of the patients, respectively. Among the 739 hospitalized patients, $146(19.8 \%)$ were classified as critical and 17 died, resulting in an in-hospital mortality rate of $2.3 \%$. Among the cohort of 739 hospitalized patients, 297 patients were identified by HCLs and 442 by PHLs. The proportion of metabolic disease in cases detected by PHLs was modestly higher than that in cases detected by HCLs (7.9\% VS 4.4\%), but the difference is not significant $(\mathrm{P}=0.056)$ (Table 1$)$. The symptoms of cough, white sputum and fatigue were the longest-lasting in the course of the disease, at $10.1 \pm 6.1$ days, $8.4 \pm 5.2$ days, and $7.1 \pm 4.5$ days, respectively. Cardiovascular diseases, pulmonary diseases, and allergy were the main comorbidities, and the numbers of patients with these three symptoms were $85(12.0 \%), 62(8.8 \%)$ and $58(8.4 \%)$, respectively. The numbers of patients with pneumonia, acute liver injury and acute respiratory distress syndrome were 444 (70.9\%), 127 (20.2\%) and 87 (13.9\%), respectively. (See Supplementary Table S1).

Frequency and time intervals of the primary medical activities of patients with different outcomes. For the patients classified as critical, the average time intervals from symptom onset to identification, first hospital visit to identification, and symptom onset to the initiation of antiviral therapy with oseltamivir was $5.5 \pm 4.1$ days, $4.0 \pm 3.3$ days and $5.8 \pm 4.5$ days, respectively, significantly longer than those for the non-critical 


\begin{tabular}{|l|c|c|c|c|}
\hline Variables & Dependent variables & OR (95\% CI for OR)* & PAR (\%) & P value \\
\hline \multirow{2}{*}{ Time intervals from onset to first visit (days) } & For criticals & $1.01(0.94-1.09)$ & 1.0 & 0.81 \\
\cline { 2 - 5 } & For deaths & $1.00(0.84-1.17)$ & 0 & 0.951 \\
\hline \multirow{2}{*}{ Time interval from onset to identification (days) } & For criticals & $1.09(1.03-1.16)$ & 9.0 & 0.005 \\
\cline { 2 - 5 } Time interval from onset to antiviral therapy(days) & For deaths & $1.13(1.02-1.26)$ & 13.0 & 0.020 \\
\cline { 2 - 5 } & For criticals & $1.12(1.03-1.22)$ & 12.0 & 0.008 \\
\hline \multirow{2}{*}{ Time interval from identification to antiviral therapy (days) } & For deaths & $1.11(0.99-1.26)$ & 11.0 & 0.086 \\
\cline { 2 - 5 } & For criticals & $0.82(0.41-1.63)$ & - & 0.57 \\
\hline \multirow{2}{*}{ Course of antiviral therapy (days) } & For deaths & $0^{\ddagger}$ & $/$ & 0.997 \\
\cline { 2 - 5 } & For criticals & $1.08(0.97-1.21)$ & 8.0 & 0.161 \\
\hline
\end{tabular}

Table 2. Multiple logistic regression for analysing the relationship of prognosis with a delay in identification and the initiation of antiviral therapy with oseltamivir. ${ }^{\star} \mathrm{OR}$ indicates the odds with each per day increase. ${ }^{\ddagger}$ The majority of patients were prescribed antiviral therapy (oseltamivir) immediately after being identified, i.e., within approximately $24 \mathrm{~h}$.

patients ( $4.4 \pm 3.1$ days, $2.8 \pm 3.6$ days and $4.3 \pm 2.9$ days, respectively). The frequency of hospital visits $(1.9 \pm 1.0$ times) for the patients with critical cases was significantly higher than that ( $1.6 \pm 1.1$ times) for the patients with non-critical cases. The average time intervals from symptom onset to identification, first hospital visit to identification, from symptom onset to the initiation of antiviral therapy with oseltamivir among the patients who died (7.6 \pm 4.7 days, $5.5 \pm 5.3$ days, $7.5 \pm 2.4$ days, respectively) were significantly longer than those among the patients who survived ( $4.5 \pm 3.7$ days, $2.5 \pm 3.8$ days and $4.5 \pm 3.7$ days, respectively) (see Supplementary Fig. S1).

A logistic regression analysis indicated that the time intervals from symptom onset to identification and from symptom onset to the initiation of antiviral therapy with oseltamivir greatly affected the number of critical case and the mortality rate of $\mathrm{pH} 1 \mathrm{~N} 1$ influenza. That is, a delay of $24 \mathrm{~h}$ in case identification led to a $9 \%$ increase in the odds of patients being classified as critical and a 13\% increase in the odds of dying, with odds ratios (ORs) of 1.09 (95\%CI: $1.03-1.16, \mathrm{P}<0.01)$ and 1.13 (95\%CI: 1.02-1.26, P < 0.05), respectively, per $24 \mathrm{~h}$ increase in the time between symptom onset and identification. A delay of $24 \mathrm{~h}$ in initiating antiviral therapy with oseltamivir led to a $12 \%$ increase in the odds of patients being classified as critical and an $11 \%$ increase in the odds of dying, with ORs of 1.12 (95\%CI: $1.03-1.22, \mathrm{P}<0.01$ ) and 1.11 (95\% CI: $0.99-1.26, \mathrm{P}=0.086)$, respectively, per $24 \mathrm{~h}$ increase in the time between symptom onset and the initiation of antiviral therapy, though the latter was not significant (Table 2).

The outcomes of pH1N1 influenza patients with different time intervals between symptom onset and case identification. The basic conditions of the three groups were comparable. However, for the patients identified more than 5 days after symptom onset, the time between symptom onset and hospital admission, the time between onset and the initiation of antiviral therapy, the length of the hospital stay and the course of the disease were significantly longer than those of patients identified within 3-5 days of symptom onset, and these time periods for the patients identified within 3-5 days of symptom onset were also longer than those for patients identified within 2 days of onset. The incidence rates of complications, such as pneumonia, acute respiratory distress syndrome, liver injury and multiple organ failure, were significantly higher among patients identified more than 5 days after symptom onset than those of patients identified within 3-5 days. The rates of the latter group were also significantly higher than those of patients identified within 2 days of symptom onset (Table 3 ). Eleven $(4.7 \%)$ of the patients identified more than 5 days after symptom onset, 3 of the patients identified within $3-5$ days and $3(1.2 \%)$ of the patients identified within 2 days (1.2\%) died. The in-hospital mortality rate of the patients identified more than 5 days later after symptom onset was significantly higher than those of the patients identified within 3-5 days and within 2 days (Table 3 ).

The outcomes of pH1N1 influenza patients with different time intervals between symptom onset and the initiation of antiviral therapy. The incidence rate of critical cases, length of the hospital stay, course of disease and the incidence rate of complications such as pneumonia, liver injury and multiple organ failure were also higher among patients who began antiviral therapy more than 5 days after symptom onset than those of patients treated within 3-5 days. These metrics were also higher for the patients treated within 3-5 days than those of patients who received antiviral therapy within 2 days of symptom onset (Table 4$)$. Six (3.4\%) of the patients who began antiviral therapy more than 5 days after symptom onset, 4 (1.7\%) of the patients who started antiviral therapy within 3-5 days, and none of patients who initiated antiviral therapy within 2 days died. The in-hospital mortality rate among patients initiating antiviral therapy more than 5 days after symptom onset was significantly higher than that of patients initiating antiviral therapy within 3-5 days. The mortality rate for the patients who began antiviral therapy within 3-5 days was also significantly higher than that of patients initiating antiviral therapy within 2 days of symptom onset (Table 4 ).

Time intervals between the primary medical activities and the outcomes of pH1N1 influenza patients with PHL-based detection and HCL-based detection. The basic conditions of the patients identified by PHLs and HCLs were very similar. However, among patients identified by HCLs, the time intervals between symptom onset and case identification, first hospital visit and case identification, and symptom onset 


\begin{tabular}{|c|c|c|c|c|}
\hline Variables & $\begin{array}{l}\text { Patient identified within } 2 \\
\text { days from onset }(\mathrm{n}=241)\end{array}$ & \begin{tabular}{|c|} 
Patient identified within \\
3-5 days from onset (257)
\end{tabular} & $\begin{array}{l}\text { Patient identified later than } \\
5 \text { days from onset (234) }\end{array}$ & Pvalue \\
\hline \multicolumn{5}{|l|}{ Base conditions } \\
\hline Gender-male n (\%) & $130(53.9)$ & $137(53.3)$ & $134(57.3)$ & 0.645 \\
\hline \multicolumn{5}{|l|}{ Age } \\
\hline $0-$ & $60(25.1)$ & $74(28.8)$ & $59(25.2)$ & \multirow{3}{*}{0.876} \\
\hline $14-$ & $161(67.4)$ & $163(63.4)$ & $156(66.7)$ & \\
\hline $60-$ & $18(7.5)$ & $20(7.8)$ & $19(8.1)$ & \\
\hline \multicolumn{5}{|l|}{ BMI } \\
\hline$<18.5$ & $57(25.9)$ & $70(29.8)$ & $55(26.6)$ & \multirow{4}{*}{0.168} \\
\hline $18.5-$ & $123(55.9)$ & $109(46.4)$ & $98(47.3)$ & \\
\hline $25-$ & $24(10.9)$ & $38(16.2)$ & $41(19.8)$ & \\
\hline $30-$ & $16(7.3)$ & $18(7.7)$ & $13(6.3)$ & \\
\hline Pulmonary disease $\mathrm{n}(\%)$ & $22(9.6)$ & $20(8.1)$ & $19(8.5)$ & 0.836 \\
\hline Cardiovascular disease n (\%) & $21(9.2)$ & $29(11.8)$ & $35(15.5)$ & 0.117 \\
\hline Metabolic disease $\mathrm{n}(\%)$ & $7(3.1)$ & $19(7.7)$ & $13(5.8)$ & 0.077 \\
\hline Renal disease $\mathrm{n}(\%)$ & $9(3.9)$ & $8(3.2)$ & $7(3.1)$ & 0.882 \\
\hline Liver disease n (\%) & $11(4.8)$ & $17(6.9)$ & $23(10.2)$ & 0.082 \\
\hline Cancers n (\%) & $5(2.2)$ & $6(2.4)$ & $5(2.2)$ & 0.983 \\
\hline $\begin{array}{l}\text { Immunosuppression disease } \\
\mathrm{n}(\%)\end{array}$ & $3(1.3)$ & $3(1.2)$ & $5(2.2)$ & 0.653 \\
\hline Nervous system disease $\mathrm{n}(\%)$ & $5(2.2)$ & $4(1.6)$ & $4(1.8)$ & 0.892 \\
\hline Pregnancy n (\%) & $12(5.2)$ & $14(5.6)$ & $16(7.1)$ & 0.679 \\
\hline \multicolumn{5}{|l|}{ Complications and Outcomes } \\
\hline $\begin{array}{l}\text { Time interval from onset to } \\
\text { hospital admission (Days, } \\
\text { mean } \pm \text { SD) }\end{array}$ & $1.2 \pm 1.1$ & $3.2 \pm 1.6$ & $6.0 \pm 3.6$ & $<0.001$ \\
\hline $\begin{array}{l}\text { Time interval from onset to } \\
\text { initiating antiviral therapy } \\
\text { (Days, mean } \pm \text { SD) }\end{array}$ & $2.1 \pm 1.6$ & $4.2 \pm 1.4$ & $7.6 \pm 3.7$ & $<0.001$ \\
\hline $\begin{array}{l}\text { Length of stay in hospital (Days, } \\
\text { mean } \pm \text { SD) }\end{array}$ & $9.7 \pm 4.8$ & $13.4 \pm 6.6$ & $17.6 \pm 9.1$ & $<0.001$ \\
\hline $\begin{array}{l}\text { Course of disease (Days, } \\
\text { mean } \pm \text { SD) }\end{array}$ & $7.5 \pm 3.6$ & $9.6 \pm 6.0$ & $11.1 \pm 6.6$ & $<0.001$ \\
\hline Pneumonia n (\%) & $102(48.3)$ & $170(78.7)$ & $166(86.5)$ & $<0.001$ \\
\hline $\begin{array}{l}\text { Acute respiratory distress } \\
\text { syndrome } \mathrm{n}(\%)\end{array}$ & $17(8.1)$ & $24(11.1)$ & $45(23.3)$ & $<0.001$ \\
\hline Liver injury n (\%) & $33(15.6)$ & $34(15.7)$ & $57(29.2)$ & 0.001 \\
\hline Renal injury n (\%) & $9(4.3)$ & $16(7.4)$ & $13(6.7)$ & 0.355 \\
\hline $\begin{array}{l}\text { Disseminated intravascular } \\
\text { coagulation } \mathrm{n}(\%)\end{array}$ & $2(1.0)$ & $1(0.5)$ & $2(1.0)$ & 0.760 \\
\hline Septic shock n (\%) & $2(0.9)$ & $7(3.2)$ & $8(4.1)$ & 0.093 \\
\hline $\begin{array}{l}\text { Nervous system complications } \\
\mathrm{n}(\%)\end{array}$ & $5(2.4)$ & $8(3.7)$ & $9(4.6)$ & 0.456 \\
\hline Multiple organ failure n (\%) & $5(2.4)$ & $10(4.6)$ & $18(9.2)$ & 0.008 \\
\hline Critical cases n (\%) & $31(12.9)$ & $47(18.3)$ & $64(27.4)$ & $<0.001$ \\
\hline In-hospital mortality n (\%) & $3(1.2)$ & $3(1.2)$ & $11(4.7)$ & 0.020 \\
\hline
\end{tabular}

Table 3. Time intervals between symptom onset and case identification and the outcomes of patients with pH1N1 influenza.

and hospital admission and the lengths of the hospital stay and the course of disease were $3.9 \pm 5.0$ days, $2.1 \pm 2.9$ days, $3.2 \pm 3.1$ days, $7.8 \pm 5.7$ days and $11.1 \pm 7.8$ days, respectively; while among patients identified by PHLs, the time intervals between symptom onset and case identification, first hospital visit and case identification, and symptom onset and hospital admission and the length of the hospital stay and the course of disease were $4.8 \pm 3.2$ days, $3.0 \pm 3.4$ days, $3.7 \pm 3.0$ days, $10.2 \pm 5.4$ days and $14.7 \pm 6.8$ days, respectively. These lengths of time for patients identified by HCLs were significantly shorter than those for patients by PHLs $(\mathrm{P}<0.01,0.05,0.01$ and 0.01 , respectively). The frequency of hospital visits ( $1.5 \pm 0.9$ times) among patients identified by HCLs was also significantly lower than that of patients identified by PHLs $(1.8 \pm 1.2$ times $)(\mathrm{P}<0.01)$ (Fig. 1$)$. Due to HCL-based detection, the frequency of hospital visits, the time intervals between symptom onset and case identification, first hospital visit and case identification, and symptom onset to hospital admission and the lengths of the hospital stay and course of disease decreased by $16.7 \%, 18.8 \%, 30.0 \%, 13.5 \%, 23.5 \%$ and $24.5 \%$, respectively (Table 1 ). Three (1.0\%) patients identified by HCLs and $14(3.2 \%)$ patients identified by PHLs died. The in-hospital mortality rate 


\begin{tabular}{|c|c|c|c|c|}
\hline Variables & $\begin{array}{l}\text { Antiviral therapy initiating within } \\
2 \text { days from onset }(n=106)\end{array}$ & $\begin{array}{l}\text { Antiviral therapy initiating in } \\
3-5 \text { days from onset }(n=229)\end{array}$ & $\begin{array}{l}\text { Antiviral therapy initiating more } \\
\text { than } 5 \text { days from onset }(n=176)\end{array}$ & P value \\
\hline \multicolumn{5}{|l|}{ Base conditions } \\
\hline Gender-male n (\%) & $57(53.8)$ & $111(48.5)$ & $105(59.7)$ & 0.082 \\
\hline \multicolumn{5}{|l|}{ Age } \\
\hline $0-$ & $29(27.4)$ & $52(22.7)$ & $42(23.9)$ & \multirow{3}{*}{0.787} \\
\hline $14-$ & $66(62.3)$ & $159(69.4)$ & $119(67.6)$ & \\
\hline $60-$ & $11(10.4)$ & $18(7.9)$ & $15(8.5)$ & \\
\hline \multicolumn{5}{|l|}{ BMI } \\
\hline$<18.5$ & $23(22.8)$ & $55(26.4)$ & $45(27.8)$ & \multirow{4}{*}{0.690} \\
\hline $18.5-$ & $54(53.5)$ & $106(51.0)$ & $80(49.4)$ & \\
\hline $25-$ & $14(13.9)$ & $31(14.9)$ & $29(17.9)$ & \\
\hline $30-$ & $10(9.9)$ & $16(7.7)$ & $8(4.9)$ & \\
\hline Pulmonary disease n (\%) & $14(13.3)$ & $22(9.6)$ & $14(8.0)$ & 0.369 \\
\hline $\begin{array}{l}\text { Cardiovascular disease } \\
\mathrm{n}(\%)\end{array}$ & $15(14.3)$ & $24(10.6)$ & $30(17.0)$ & 0.165 \\
\hline Metabolic disease n (\%) & $7(6.7)$ & $15(6.6)$ & $9(5.1)$ & 0.797 \\
\hline Renal disease $\mathrm{n}(\%)$ & $5(4.8)$ & $8(3.5)$ & $6(3.5)$ & 0.839 \\
\hline Liver disease n (\%) & $8(7.6)$ & $17(7.5)$ & $17(9.7)$ & 0.697 \\
\hline Cancers n (\%) & $1(1.0)$ & $7(3.1)$ & $4(2.3)$ & 0.444 \\
\hline $\begin{array}{l}\text { Immunosuppression disease } \\
\mathrm{n}(\%)\end{array}$ & $3(2.9)$ & $4(1.8)$ & $3(1.7)$ & 0.784 \\
\hline $\begin{array}{l}\text { Nervous system disease } \\
\mathrm{n}(\%)\end{array}$ & $2(1.9)$ & $4(1.7)$ & $3(1.7)$ & 0.992 \\
\hline Pregnancy n (\%) & $6(5.7)$ & $15(6.6)$ & $10(5.7)$ & 0.919 \\
\hline \multicolumn{5}{|l|}{ Complication and Outcomes } \\
\hline $\begin{array}{l}\text { Length of stay in hospital } \\
\text { (Days, mean } \pm \text { SD) }\end{array}$ & $8.3 \pm 3.7$ & $9.0 \pm 5.2$ & $10.9 \pm 6.5$ & 0.001 \\
\hline $\begin{array}{l}\text { Course of disease (Days, } \\
\text { mean } \pm \text { SD) }\end{array}$ & $11.5 \pm 5.1$ & $12.3 \pm 5.6$ & $17.0 \pm 7.1$ & $<0.001$ \\
\hline Pneumonia n (\%) & $68(74.7)$ & $152(74.9)$ & $146(88.0)$ & 0.003 \\
\hline $\begin{array}{l}\text { Acute respiratory distress } \\
\text { syndrome } \mathrm{n}(\%)\end{array}$ & $12(13.2)$ & $28(13.7)$ & $33(20.0)$ & 0.195 \\
\hline Liver injury n (\%) & $21(23.1)$ & $30(14.6)$ & $49(29.3)$ & 0.002 \\
\hline Renal injury n (\%) & $5(5.5)$ & $12(5.9)$ & $14(8.4)$ & 0.556 \\
\hline $\begin{array}{l}\text { Disseminated intravascular } \\
\text { coagulation } \mathrm{n}(\%)\end{array}$ & $0(0.0)$ & $1(0.5)$ & $2(1.2)$ & 0.385 \\
\hline Septic shock n (\%) & $4(4.4)$ & $5(2.4)$ & $7(4.2)$ & 0.554 \\
\hline $\begin{array}{l}\text { Nervous system } \\
\text { complications n (\%) }\end{array}$ & $0(0.0)$ & $7(3.4)$ & $6(3.6)$ & 0.055 \\
\hline Multiple organ failure n (\%) & $3(3.3)$ & $7(3.4)$ & $16(9.6)$ & 0.025 \\
\hline Critical cases n (\%) & $20(18.9)$ & $41(17.9)$ & $49(27.8)$ & 0.045 \\
\hline $\begin{array}{l}\text { In-hospital mortality } \\
\text { (crude) }\end{array}$ & $0(0.0)$ & $4(1.7)$ & $6(3.4)$ & 0.043 \\
\hline
\end{tabular}

Table 4. Time intervals between symptom onset and the initiation of antiviral therapy and the outcomes of patients with pH1N1 influenza.

of the former was significantly lower than that of the latter $(\mathrm{P}<0.05)$. With HCL-based detection, the in-hospital mortality rate decreased by $68.8 \%$.

Detection of the pH1N1 influenza virus in different hospitals. Of the 65 hospitals whose data were available, $29.0 \%$ (18) were upper first-class hospitals, 37.1\% (23) were middle first-class hospitals, 25.8\% (16) were upper second-class hospitals, and $8.1 \%$ (5) were middle second-class hospitals. The mortality rates varied in different hospital levels. Of the 65 hospitals, only 13 (20.0\%) could conduct the methods required for pH1N1 viral detection, such as RT-PCR and rtRT-PCR detection, during the 2009 pandemic. Among these 13 hospitals, results from 6 needed to be rechecked by the local Center for Disease Control and Prevention. Fifty-two hospitals (80\%) could not conduct $\mathrm{pH} 1 \mathrm{~N} 1$ viral detection, and their specimens were sent to the local Center for Disease Control and Prevention for detection. The reasons why hospitals could not conduct $\mathrm{pH} 1 \mathrm{~N} 1$ viral detection included prohibition by local authorities and hospitals themselves (80\%) and the lack of funds (21.5\%), instruments (20.0\%) and training (18.5\%). Of the 65 hospitals, 40 (61.5\%) were less than 10 kilometres from the local Center for Disease Control and Prevention, 15 (23.1\%) were within 10-60 kilometres, and 3 (4.6\%) were more than 60 kilometres away (see Supplementary Table S2). 


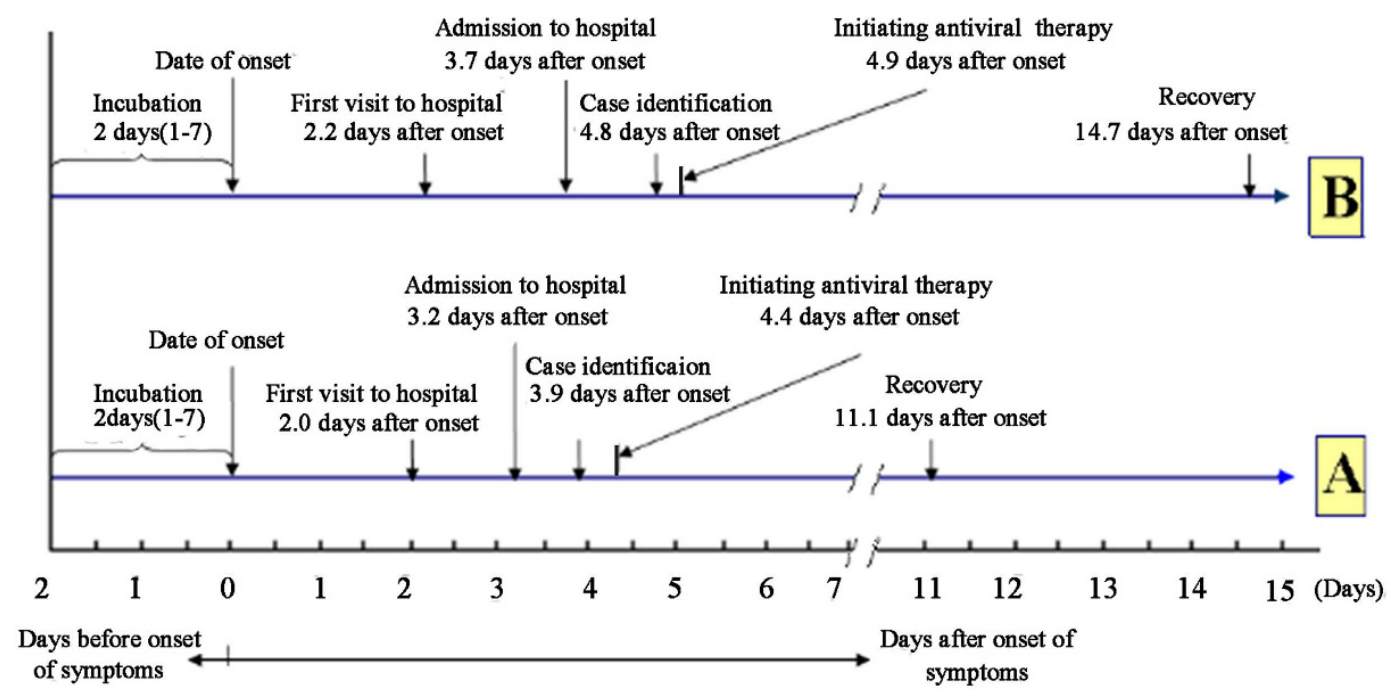

Figure 1. The timetable of major medical activities in the course of infection with pH1N1 influenza. (A) Patients in hospitals that could detect the $\mathrm{pH} 1 \mathrm{~N} 1$ virus. (B) Patients in hospitals that could not detect the pH1N1 virus.

\section{Discussion}

Though the H1N1 pandemic has ended, the $2009 \mathrm{pH} 1 \mathrm{~N} 1$ influenza outbreak has left us with numerous lessons to summarize and learn. Our findings indicated that a delay of $24 \mathrm{~h}$ in case identification led to a $10 \%$ increase in the odds of a patient being classified as critical. In severe $\mathrm{pH} 1 \mathrm{~N} 1$ influenza cases, patients generally begin to deteriorate around 3 to 5 days after symptom onset ${ }^{10,11}$. Due to the delayed identification, the incidence rates of complications such as pneumonia, acute respiratory distress syndrome, liver injury and multiple organ failure were significantly increased. A delay of $24 \mathrm{~h}$ in identification led to a $13 \%$ increase in odds of dying. The average time intervals between symptom onset and identification, first hospital visit and identification, and symptom onset and the initiation of antiviral therapy with oseltamivir among patients who died were longer than those among patients who survived. The in-hospital mortality rate of patients identified more than 5 days after symptom onset was significantly higher than those of patients identified within 3-5 days and within 2 days. A study by Echevarría-Zuno indicated that delayed identification was associated with delayed admission (with an OR of 1.19 per day $)^{12}$. However, our findings indicated that delayed identification not only resulted in delayed admission but also led to delayed antiviral therapy, critical classification and even death.

Many studies assessing the difference in the in-hospital mortality rate between patients given antiviral therapy within 2 days of symptom onset and those started on antiviral therapy 2 days later indicated that the early use of antivirals could also effectively decrease the in-hospital mortality rate ${ }^{13-15}$. In those beyond 2 days of symptom onset who are moderately or severely ill, antivirals could still be beneficial ${ }^{16-18}$. Vernon J. Lee and co-workers provided evidence that early case detection and the use of antiviral ring prophylaxis effectively truncated the spread of infection during an epidemic ${ }^{19}$. Our findings further strengthened the conclusion that delayed antiviral therapy with oseltamivir could increase the in-hospital mortality rate, lengths of the hospital stay and course of disease, and the incidence rates of critical cases and complications, in particular pneumonia, liver injury and multiple organ failure. A delay of $24 \mathrm{~h}$ in beginning antiviral therapy with oseltamivir led to a $12 \%$ increase in odds of patients being classified as critical. The in-hospital mortality rate among patients who began antiviral therapy more than 5 days after symptom onset was significantly higher than that of patients who began antiviral therapy within 3-5 days. The in-hospital mortality rate of patients who began treatment within 3-5 days was also significantly higher than that of patients initiating antiviral therapy within 2 days of symptom onset. The large number of patients in our study, which enabled us to assess the differences in in-hospital mortality rates among patients who received antiviral therapy within 2 days, within 3-5 days and more than 5 days after symptom onset, indicated that effect of antiviral therapy initiated within 3-5 days was better (or at least significant) than that initiated more than 5 days after symptom onset, though it was not as good as antiviral therapy initiated within 2 days.

Early identification and good patient compliance could improve early antiviral therapy. Our results indicated that a majority of the patients treated with oseltamivir immediately (within approximately $24 \mathrm{~h}$ ) after being identified exhibited good compliance, but antiviral therapy could be postponed by delayed identification. In fact, the prerequisite for early admission and early antiviral therapy is early case identification. Measures such as developing better diagnostic methods ${ }^{20}$, raising the diagnostic awareness of physicians, and simplifying the workflow of patients visiting hospitals might increase the early identification of patients. The model of HCL-based detection could make detection more convenient and enhance diagnostic abilities and thus facilitate early case identification. Our study indicated that, due to HCL-based detection, the frequency of patients' hospital visits, the time intervals between symptom onset and case identification, first hospital visit and case identification, and symptom onset to hospital admission, and the lengths of their hospital stay and course of disease decreased by $16.7 \%$, $18.8 \%, 30.0 \%, 13.5 \%, 23.5 \%$ and $24.5 \%$, respectively. 
According the Law on Prevention and Control of Infectious Diseases ${ }^{21}$, PHLs have an obligation to confirm every suspected case of infectious disease. Some hospitals, considering it a requirement of treatment, are able to detect infectious disease cases. However, during the 2009 pandemic, a low proportion (only $20.0 \%$ ) of the hospitals were able to perform $\mathrm{pH} 1 \mathrm{~N} 1$ viral detection. The results from $50 \%$ of these hospitals needed to be rechecked by the local Center for Disease Control and Prevention. The main reason for the low proportion of hospitals able to perform $\mathrm{pH} 1 \mathrm{~N} 1$ viral detection was that it is not supported by hospitals themselves. Though the Ministry of Health of the People's Republic of China issued a notice on December 7, 2009, that the first-class hospitals were authorized to perform $\mathrm{pH} 1 \mathrm{~N} 1$ viral detection ${ }^{22}$, local authorities and hospitals preferred to have the detection done by the local CDC laboratories rather than the hospital clinical laboratories in order to ensure biosafety. Another reason for the low proportion was the lack of equipment, funds, staff and techniques. Unlike the detection of bacteria, which has been widely carried out in HCLs ${ }^{23,24}$, viral detection was not common in HCLs, especially for emerging viral infectious diseases as pH1N1 influenza ${ }^{25,26}$. The WHO Global Influenza Surveillance Network, one of the biggest infectious disease surveillance systems in the detection also obtained its data from PHLs rather than HCLs (the National Influenza Center and sentinel laboratories in each country) ${ }^{27,28}$.

Our study indicates that HCL-based detection could improve early identification and early antiviral therapy and thus reduce in-hospital mortality rates. The in-hospital mortality rate among patients with HCL-based detection was significantly lower than that of patients with PHL-based detection. With HCL-based detection, the in-hospital mortality rate decreased by $68.8 \%$. The Department of Infectious Disease in hospitals and community hospitals is the first line of diagnosis and treatment for $\mathrm{pH} 1 \mathrm{~N} 1$ influenza. Clinical microbiology laboratories could play a key role in the detection and identification of biological agents ${ }^{29}$. Therefore, public health preparedness and health care reform should focus on enhancing the ability of HCLs, including clinical microbiology laboratories and clinical virology laboratories, to detect biological agents. Given that the detection techniques, such as RT-PCR and rtRT-PCR test, are relatively sophisticated, they need to be popularized and enhanced in hospitals ${ }^{30}$. In particular, during the pandemic, the medical and health resources were in relatively short supply, so the HCLs are advised to take on more detection work. Influenza pandemics and epidemics of other infectious diseases are inevitable, so a public health security system should be established to respond to these epidemic or pandemics $^{31}$. A delay in identification and the initiation of antiviral therapy would have a negative effect on the prognosis of patients with $\mathrm{pH} 1 \mathrm{~N} 1$ influenza, and it could be improved by the model of HCL-based detection. This lesson, though learned from China, could be applicable to other parts of the world in the fight against epidemics or pandemics of influenza.

The measures that should be taken to enhance the ability of HCLs to detect influenza are as follows: first, the local government should increase governmental support, including equipment, special funds and staff; second, hospital administrators should understand the significance and benefits of such detection and support (or at least not prohibit) the involvement of their laboratories; and third, the coordination, communication and collaboration among PHLs and HCLs needs to be further strengthened. By enhancing the ability of HCLs to detect influenza, hospitals would benefit from improved education and collaboration with PHLs, which in turn would result in faster identification of outbreaks and better patient outcomes ${ }^{32,33}$.

Undeniably, there were limitations to our study. There was a potential lead-time bias in this study. Severe or critical patients would visit the hospital earlier and draw more attention from physicians. Even if early treatment had no benefit, the time interval between symptom onset and first hospital visit is shorter simply by the addition of the lead time ${ }^{34}$. Second, though the level of hospitals was the same, a hospital with the ability to detect influenza virus, compared to one without this ability, would have more favourable conditions for the treatment of influenza. In addition, though the levels of hospitals the patients with PHL- and HCL-based detection visited were comparable, no specific and detailed methods were taken to assess and compare the treatment capacities of the two groups of hospitals in this study.

\section{Methods}

The case sources and identification. As pH1N1 became epidemic in China, the Ministry of Public Health of China responded quickly, organizing experts to compile the pH1N1 2009 Clinical Guidelines $\left(1^{\text {st }}-3^{\text {rd }}\right.$ Edition, 2009) ${ }^{35-37}$, and made an official announcement proclaiming that the diagnosis and treatment of pH1N1 should be conducted in accordance with the guidelines. Hospitalized patients were tracked, and their information was recorded by sentinel investigators. Daily respiratory tract specimens were collected from patients during their hospitalization and tested by real-time RT-PCR to detect the nucleic acid of the $2009 \mathrm{pH} 1 \mathrm{~N} 1$ virus. According the Law on Prevention and Control of Infectious Diseases ${ }^{21}$, PHLs are authorized to obtain any suspected infectious disease samples from any hospital. Therefore, the PHLs and HCLs had the same opportunities to obtain samples.

The diagnosis of cases and classification of cohorts. The confirmed cases of $\mathrm{pH} 1 \mathrm{~N} 1$ virus infection were defined as any cases confirmed by the Chinese CDC. According to the pH1N1 2009 Clinical Guidelines $\left(1^{\text {st }}-3^{\text {rd }}\right.$ Edition, 2009) released by the Chinese $\mathrm{MOH}^{35-37}$, critical cases were defined as any cases with following symptoms at admission: (1) respiratory failure; (2) septic shock; (3) multiple organs insufficiency; and (4) other critical clinical conditions requiring intensive care. For each patient, we calculated the time between symptom onset and first visit, symptom onset and identification (laboratory confirmed), first hospital visit to identification, symptom onset to the initiation of antiviral therapy, and identification and the initiation of antiviral therapy, as well as length of antiviral therapy time.

A total of 12,894 cases of influenza were reported between May 1 and December 21, 2009, in Zhejiang, China. We planned to randomly investigate 800 cases and were finally able to collect 739 valid cases for our cohort, which accounted for approximately $5.7 \%$ of the total number of cases. The cohort was developed by collecting clinical data and laboratory samples. The cohort was divided into two groups according to the different types of 
laboratories by which their samples were detected: one group was identified by PHLs and the other by HCLs. The cohort was also divided into three subgroups according to the time interval between symptom onset to identification: within 2 days of onset (identification $\leq 2$ days); 3-5 days after onset (identification 3-5 days); more than 5 days after onset (identification $>5$ days). Antiviral therapy was defined as a therapy with at least 1 day of oseltamivir treatment. The cohort was also divided into three subgroups according to the timeliness of oseltamivir administration: within 2 days after illness onset (oseltamivir $\leq 2$ days); $3-5$ days after onset (oseltamivir 3-5 days); more than 5 days after onset (oseltamivir $>5$ days). We attempted to control for the admission rate bias in this study using a stratification based on the different level of hospitals. Apart from the differences in patients with pH1N1 influenza detected by HCLs or PHLs, the various cases of pH1N1 influenza were randomly distributed to different hospitals, and two groups of patients, separately identified by HCLs and PHLs, were demographically comparable on the presumption that the stratification was based on the different hospital levels.

Antiviral and symptomatic therapy of pH1N1 influenza. According to the protocol for the diagnosis and treatment of pH1N1 influenza ( $1^{\text {st }}-3^{\text {rd }}$ Edition, 2009), antiviral therapy would be used to treat the severe and critical cases and high risk cases infected with influenza A pandemic (H1N1) virus. For adults, oseltamivir was prescribed according to the standard dosing regimen ( $75 \mathrm{mg}$ twice daily orally for 5 days), and a dosage adjustment would be made for critical cases with a dosing regimen ( $150 \mathrm{mg}$ twice daily orally for 5 days). For children, a dosage adjustment was made according to their body weight (BW, $30 \mathrm{mg}$ Bid for children with BW $<15 \mathrm{~kg} ; 45 \mathrm{mg}$ Bid for BW 15-23 kg; $60 \mathrm{mg}$ Bid for BW 23-40 kg; and $75 \mathrm{mg}$ Bid for BW $>40 \mathrm{~kg}$ ).

According to the protocol for the diagnosis and treatment of pH1N1 influenza (1st-3rd Edition, 2009), the appropriate antimicrobial and/or antifungal agents would be administered when patients presented with combined bacterial and/or fungal infections. Patients with hypoxemia or respiratory failure should promptly be given the appropriate oxygen therapy or mechanical ventilation, and patients with combined shock should be given the corresponding anti-shock treatment. In the cohorts, except for oseltamivir, the frequency of administration of other treatments, including antibiotics, traditional Chinese medicine and oxygen therapy, was similar between groups $(\mathrm{P}>0.05)$.

Data collection. The information was collected in pre-defined standard data collection forms. The investigation, being part of the national database and coordinated by the Chinese Ministry of Health, took place in Zhejiang province, and further information was added about the mode of case identification. The data collection forms were filled in by the hospital sentinels. The sentinel investigators were primarily infectious diseases physicians, closely involved in the care of the patients at their centres. Systematic training was conducted for all sentinel investigators (infectious diseases physicians), including a course covering data collection and methods of detection, diagnosis, treatment and follow-up for $\mathrm{pH} 1 \mathrm{~N} 1$ influenza. The information collected from the patients was as follows: symptom onset, comorbidities, contact history, date of primary medical onset, visit and admission to hospital(including the frequency of hospital visits: the number of times a patient visited the hospital for outpatient treatment before admission), the types of laboratories by which patients' samples were detected, antiviral therapy, complications, duration of the viral shedding, prognosis and outcomes. Hospital information, including the hospital class and level, the distance from the hospital to the local CDC, the cooperation between hospitals and the CDC, the ability of the hospital to detect the pH1N1 influenza virus and the reasons why the hospitals could not detect the $\mathrm{pH} 1 \mathrm{~N} 1$ virus were collected and taken into consideration. The data of collecting main factors for analysis had a low missing rate (less than $7.0 \%$ ).

Statistical analysis. The demographic, clinical and laboratory characteristics, time intervals between onset and primary medical activities and outcomes was reported and analysed. Means (standard deviations, SD) or medians (interquartiles, IQR) were calculated as summaries of continuous variables, and incidence numbers (percentage) were calculated as summaries of categorical variables. We compared different demographic and clinical features, time intervals of main medical activities and outcomes by an ANOVA test, chi-square test, or Fisher's exact test, as appropriate. The risk factor analysis of the independent variables was conducted with a multiple stepwise logistic regression model, in which $\mathrm{P}=0.10$ was given for the entering level, and $\mathrm{P}=0.15$ for the excluding level. The testing level was given $\mathrm{a}=0.05$. The variables used for the multiple logistic regression analysis included the time interval from onset to first visit (days), time interval from onset to identification (days), time interval from onset to the initiation of antiviral therapy (days), time interval from identification to the initiation of antiviral therapy (days) and course of antiviral therapy (days). The data were put into a database in duplicate by different operators, and all the analyses were conducted using SAS version 9.3. Figure 1 was processed by GIMP 2.

Ethics Statement. The research ethics board at First Affiliated Hospital, School of Medicine, Zhejiang University approved the design and procedure of this study. All methods were performed in accordance with the relevant guidelines and regulations. Informed consent was obtained from all patients.

\section{References}

1. Laguna-Torres, V. A. \& Benavides, J. G. Infection and death from influenza A H1N1 virus in Mexico. LANCET 374, 2032 (2009).

2. Dawood, F. S. et al. Emergence of a novel swine-origin influenza A (H1N1) virus in humans. N Engl J Med 360, 2605 (2009).

3. Smith, G. J. et al. Dating the emergence of pandemic influenza viruses. Proc Natl Acad Sci USA 106, 11709 (2009).

4. Pan, C. et al. Genomic signature and mutation trend analysis of pandemic (H1N1) 2009 influenza A virus. Plos One 5, e9549 (2010).

5. Khan, K. et al. Spread of a novel influenza A (H1N1) virus via global airline transportation. N Engl J Med 361, 212 (2009).

6. Pandemic (H1N1) 2009 - update 94. Geneva: World Health Organization, April 1, 2010.Available at http://www.who.int/csr/ don/2010_04_01/en/index.html (2010). 
7. Gao, R. et al. Development and implementation of the quality control panel of RT-PCR and real-time RT-PCR for avian influenza A (H5N1) surveillance network in mainland China. BMC INFECT DIS 11, 67 (2011).

8. CDC protocol of realtime RTPCR for swine influenza A(H1N1):World Health Organization,28 April 2009.Available at:www.who.

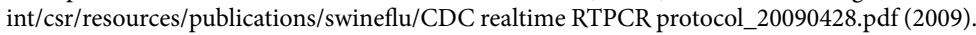

9. Astles, J. R., White, V. A. \& Williams, L. O. Origins and development of the National Laboratory System for public health testing. PUBLIC HEALTH REP 125, Suppl 218 (2010).

10. World Health Organization, Clinical features of severe cases of pandemic influenza. Available at http://www.who.int/csr/disease/ swineflu/notes/h1n1_clinical_features_20091016/en/ (2009).

11. Zhao, C., Gan, Y. \& Sun, J. Radiographic study of severe Influenza-A (H1N1) disease in children. Eur J Radiol 79, 447 (2011).

12. Echevarria-Zuno, S. et al. Infection and death from influenza A H1N1 virus in Mexico: a retrospective analysis. Lancet 374, 2072 (2009).

13. Slopen, M. E. et al. Patients hospitalized with 2009 pandemic influenza A (H1N1) - New York City, May 2009. MMWR Morb Mortal Wkly Rep 58, 1436 (2010).

14. Dominguez-Cherit, G. et al. Critically Ill patients with 2009 influenza A(H1N1) in Mexico. Jama 302, 1880 (2009).

15. Jamieson, D. J. et al. H1N1 2009 influenza virus infection during pregnancy in the USA. LANCET 374, 451 (2009).

16. Yang, S. G. et al. Antiviral therapy and outcomes of patients with pneumonia caused by influenza A pandemic (H1N1) virus. Plos One 7, e29652 (2012).

17. Viasus, D. et al. Timing of oseltamivir administration and outcomes in hospitalized adults with pandemic 2009 influenza A(H1N1) virus infection. Chest 140, 1025 (2011).

18. McGeer, A. et al. Antiviral therapy and outcomes of influenza requiring hospitalization in Ontario, Canada. Clin Infect Dis 45, 1568 (2007).

19. Lee, V. J. et al. Oseltamivir ring prophylaxis for containment of $2009 \mathrm{H} 1 \mathrm{~N} 1$ influenza outbreaks. N Engl J Med 362, 2166 (2010).

20. Kandun, I. N. et al. Factors associated with case fatality of human H5N1 virus infections in Indonesia: a case series. Lancet 372, 744 (2008).

21. Law of the People's Republic of China on Prevention and Treatment of Infectious Diseases (2004 Revision).Available at http://www. gov.cn/banshi/2005-05/25/content_971.htm (2004).

22. Ministry of Health of the People's Republic of China. Available at: http://www.moh.gov.cn/publicfiles/business/htmlfiles/mohyzs/ s3586/200912/44828.htm (2009).

23. Tissari, P. et al. Accurate and rapid identification of bacterial species from positive blood cultures with a DNA-based microarray platform: an observational study. Lancet 375, 224 (2010).

24. Basu, M. et al. Nano-biosensor development for bacterial detection during human kidney infection: use of glycoconjugate-specific antibody-bound gold NanoWire arrays (GNWA). Glycoconj J 21, 487 (2004).

25. Keim, M. E., Pesik, N. \& Twum-Danso, N. A. Lack of hospital preparedness for chemical terrorism in a major US city: 1996-2000. Prehosp Disaster Med 18, 193 (2003)

26. Nolte, K. B., Yoon, S. S. \& Pertowski, C. Medical examiners, coroners, and bioterrorism. Emerg Infect Dis 6, 559 (2000).

27. Available at: http://www.who.int/csr/disease/influenza/surveillance/en/index.html.

28. Ortiz, J. R. et al. Monitoring influenza activity in the United States: a comparison of traditional surveillance systems with Google Flu Trends. Plos One 6, e18687 (2011).

29. Klietmann, W. F. \& Ruoff, K. L. Bioterrorism: implications for the clinical microbiologist. CLIN Microbiol Rev 14, 364 (2001).

30. Balish, A. et al. Evaluation of rapid influenza diagnostic tests for detection of novel influenza A (H1N1) Virus - United States, 2009. MMWR Morb Mortal Wkly Rep 58, 826 (2009).

31. Memish, Z. A. et al. Establishment of public health security in Saudi Arabia for the 2009 Hajj in response to pandemic influenza A H1N1. Lancet 374, 178k (2009).

32. Marshall, S. A., Brokopp, C. D. \& Size, T. Leadership principles for developing a statewide public health and clinical laboratory system. Public Health Rep 125, Suppl 2110 (2010).

33. Inhorn, S. L. et al. The State Public Health Laboratory System. Public Health Rep 125, Suppl 24 (2010).

34. Richards, M. A., Westcombe, A. M., Love, S. B., Littlejohns, P. \& Ramirez, A. J. Influence of delay on survival in patients with breast cancer: a systematic review. LANCET 353, 1119 (1999).

35. Protocol for diagnosis and treatment of $2009 \mathrm{H} 1 \mathrm{N1}$ influenza (2009 first edition trial version). Available at http://www.nhfpc.gov. $\mathrm{cn} / \mathrm{yzygj} / \mathrm{s} 3585 \mathrm{u} / 200905 / \mathrm{e} 83 \mathrm{~b} 132 \mathrm{ecd} 0745 \mathrm{e} 0 \mathrm{bfd} 89 \mathrm{ec} 220 \mathrm{faa} 0 \mathrm{a} 8 . \mathrm{shtml}$.

36. Zhong, N., Li, L., Wang, C. \& Chao, E. Protocol for diagnosis and treatment of $2009 \mathrm{H} 1 \mathrm{~N} 1$ influenza (2009 second edition). National Medical Journal of China 89, 2526 (2009).

37. Protocol for diagnosis and treatment of $2009 \mathrm{H} 1 \mathrm{~N} 1$ influenza (2009 third edition).Available at http://www.nhfpc.gov.cn/yzygj/ s3593g/201306/5fc4b2d158d7475fa0da32e959f9a7ac.shtml (2009).

\section{Acknowledgements}

This study was supported by a grant from Mega-projects of Science Research for the 12th Five-Year Plan of China (2014ZX10004008, 2013ZX10004901, 2013ZX10004904, 2011ZX10004901), the National Natural Science Foundation of China $(81001271,81672005)$, the key project the National Natural Science Foundation of China and Guangdong Provincial Government (U1611264), the Fundamental Research Funds for the Central Universities and the open-research project of State Key Laboratory for Diagnosis and Treatment of Infectious Diseases, The First Affiliated Hospital, College of Medicine, Zhejiang University, China (2016KF11).

\section{Author Contributions}

Shigui Yang and Lanjuan Li planned the study. Shigui Yang, Yuqing Zhou, Yuanxia Cui, Cheng Ding, Jie Wu, Min Deng, Chencheng Wang, Xiaoqing Lu, Xiaoxiao Chen, Yiping Li, Dongyan Shi, Feng-fang Mi, and Lanjuan Li supervised the data collection, data entry, and data processing. Shigui Yang and Lanjuan Li had full access to all the study data and assume responsibility for the data integrity and the accuracy of the data analysis. Shigui Yang wrote the first draft of the paper and has primary responsibility for the final content. All authors contributed to and approved the final manuscript.

Additional Information

Supplementary information accompanies this paper at http://www.nature.com/srep

Competing Interests: The authors declare no competing financial interests. 
How to cite this article: Yang, S. et al. The need for strengthening the influenza virus detection ability of hospital clinical laboratories: an investigation of the 2009 pandemic. Sci. Rep. 7, 43433; doi: 10.1038/srep43433 (2017).

Publisher's note: Springer Nature remains neutral with regard to jurisdictional claims in published maps and institutional affiliations.

(c) (i) This work is licensed under a Creative Commons Attribution 4.0 International License. The images or other third party material in this article are included in the article's Creative Commons license, unless indicated otherwise in the credit line; if the material is not included under the Creative Commons license, users will need to obtain permission from the license holder to reproduce the material. To view a copy of this license, visit http://creativecommons.org/licenses/by/4.0/

(C) The Author(s) 2017 